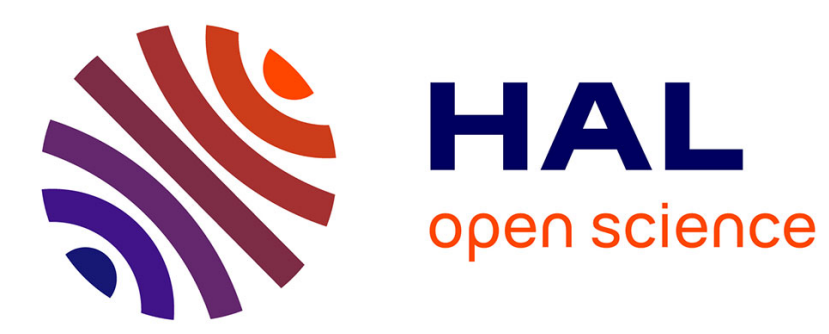

\title{
Electrical Fault Characterization by Hough Transform of Phase Diagram Information
}

Costin Vasile, Angela Digulescu, Cornel Ioana

\section{To cite this version:}

Costin Vasile, Angela Digulescu, Cornel Ioana. Electrical Fault Characterization by Hough Transform of Phase Diagram Information. COMCAS 2017 - IEEE International Conference on Microwaves, Antennas, Communications and Electronic Systems, Nov 2017, Tel-Aviv, Israel. 10.1109/COMCAS.2017.8244792 . hal-01592965

\section{HAL Id: hal-01592965 \\ https://hal.science/hal-01592965}

Submitted on 25 Sep 2017

HAL is a multi-disciplinary open access archive for the deposit and dissemination of scientific research documents, whether they are published or not. The documents may come from teaching and research institutions in France or abroad, or from public or private research centers.
L'archive ouverte pluridisciplinaire HAL, est destinée au dépôt et à la diffusion de documents scientifiques de niveau recherche, publiés ou non, émanant des établissements d'enseignement et de recherche français ou étrangers, des laboratoires publics ou privés. 


\title{
Electrical Fault Characterization by Hough Transform of Phase Diagram Information
}

\author{
C. Vasile ${ }^{1}$, A. Digulescu ${ }^{2}$, C. Ioana ${ }^{1}$ \\ ${ }^{1}$ Univ. Grenoble Alpes, GIPSA-lab, DIS, 11 rue des Mathématiques, BP 46, 38402, Saint Martin \\ d'Hères Cedex France \\ ${ }^{2}$ Military Technical Academy, 34-39 George Cosbuc Avenue, Bucharest, Romania
}

\begin{abstract}
This paper presents a solution of a well-known problem in electrical energy distribution: the detection of partial discharges in a distribution system also used for communication (such as power control signals). We show the inconveniences of the classical approach (energy thresholding) and we present the results of a new approach, based on the projection of the signals in a multidimensional phase space, followed by a Hough transform-based analysis.
\end{abstract}

Index Terms - electrical fault, partial discharge, Hough transform, phase diagram, phase space trajectory.

\section{INTRODUCTION}

The topic of fault detection in electrical system is of great interest in the present day, and one of the most common faults is represented by partial discharges (PDs). More than $30 \%$ percent of breakdowns in power systems are caused by isolation defects [1], which can occur in any phase of the production-transport-distribution chain.

From o signal signature point of view, PDs are very short, impulse-like transient signals, while also presenting a wide band frequency characteristic. From a physical point of view, the phenomenon is also associated with electromagnetic, ultrasonic and acoustic emissions. Based on these features there exist several procedures for PD detection and/or localization [2]:

- Electrical measurement-based detection/localization;

- Electromagnetic sensing;

- Acoustic/ultrasound sensing;

In the context of electrical-based detection, the classical approach is to use current sensors (Rogowski or current transformers) with a wide bandwidth and inspecting the High Frequency domain of the current $(10 \mathrm{kHz}$ and upwards to tens of $\mathrm{MHz}$ ). This approach, coupled with energy thresholding, is very robust in a non-noisy environment. But in real-life applications, there are noise sources in the system, which can interfere with proper detection. Loads also generate high frequency content, but not at the same levels as PDs and of a different temporal pattern.

The most difficult problem to overcome for detecting the temporal signature of the high frequency emissions of the PDs is identifying their presence in a network used for communications purposes, such as power control signals. These types of systems normally generate a high frequency content of levels greater than the PD and is difficult to distinguish between the two phenomena.

The method proposed in this paper attempt firstly to represent the two phenomena, the PD and communications, in the phase diagram domain that shows up good discrimination capability for the both signal types. The, the Hough transform is applied in order to measure this discrimination and to allow the accurate identification.

This paper presents the detection problem in more detail in section 2; in section 3 a new approach based on phase space embedding is explored, followed by experimental results and the concluding remarks.

\section{PRACTICAL PROBLEM FORMULATION: PDS VS COMMS}

As stated in the previous section, one of the most employed PD detection principles is electrical-based sensing, with the use of high frequency current measurements. The energy levels of the high frequency current are compared to a threshold and a decision is taken [3].

This approach fares well in a noise-free electrical system, seeing as (depending on the analyzed bandwidth) loads generate traditionally lower levels of high frequency energy.

But one of the most important high frequency noise sources are communication systems, that employ carrier frequencies spread over a wide band spectrum and thoroughly mask the PD phenomenon.

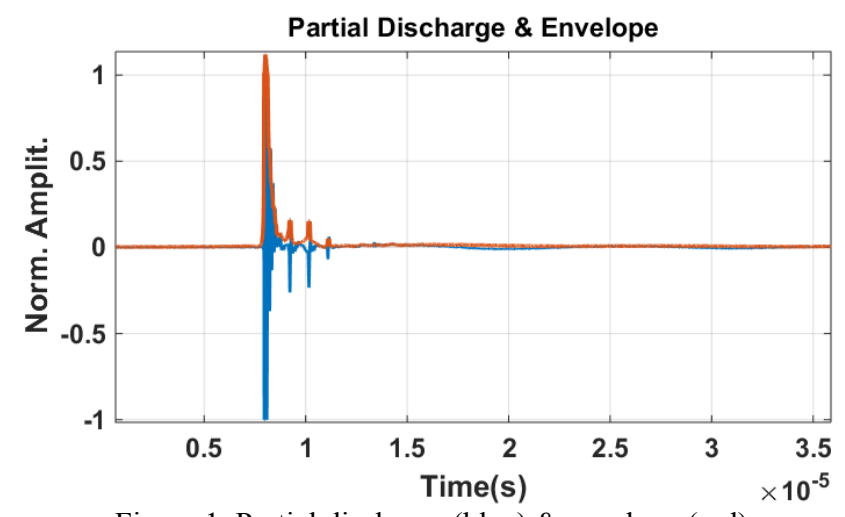

Figure 1. Partial discharge (blue) \& envelope (red).

An example of a recorded partial discharge is shown in figure 1 , which is clearly visible in a noise-less environment. Note that the signal, as all the other signals, are normalized between 0 and 1 .

Figure 2 presents the case where a PD happens during the communication of the same previous message as before, and the transient signal of interest has become a lot more difficult to recognize (green rectangle). 


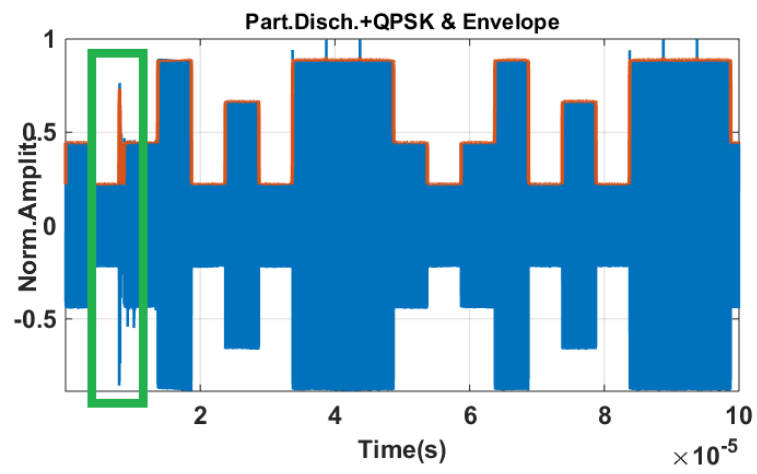

Figure 2. $\mathrm{PD}+\mathrm{COMM}$ signals (blue) $\&$ envelope (red).

Figure 3 shows the power spectral density (Welch's method) for the signal in figure 2, showing the amplitude masking of the PD phenomenon. This type of communication uses all the classical modulation techniques: amplitude, frequency and phase modulation.

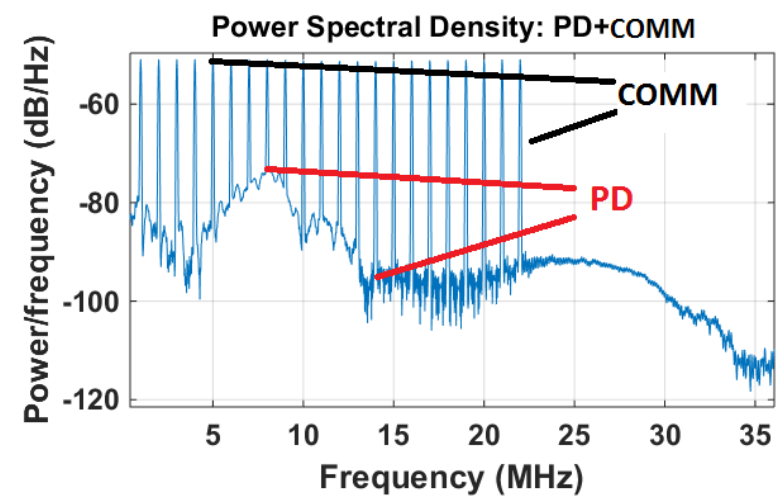

Figure 3. Power Spectral Density: PD+COMM

A different approach than a simple energy detector is required aimed to identify the presence of the partial discharge in this signal window (and others similar).

\section{PHASE SPACE-BASED ANALYSIS}

Seeing as energy detectors or matched filter-like approaches are limited in this context, we propose an approach based on the embedding in the phase space.

A measured signal can be considered as a time series $s$ of length $N$ samples, as expressed in equation (1).

$$
s=\{s[1], \ldots, s[N]\}
$$

The time series is embedded in an $m$-dimensional phase space, as described by Takens \& Abarbanel in [6], [7]. In this new representation the time series becomes a succession of $m$-dimensional vectors of the form:

$$
\overrightarrow{v_{[l]}}=\sum_{k=1}^{m} s[i+(k-1) \tau] \cdot \overrightarrow{e_{k}}, i=\overline{1, M}
$$

where $\overrightarrow{v_{[l]}}$ are the vectors, $m$ is the embedding dimension, $\tau$ is the lag (delay) taken between samples, $M=N-(m-1)$ and $\overrightarrow{e_{k}}$ are the vectors of the unit axis. By computing the phase space trajectory, we can completely unfold the dynamics of the phenomena under inspection.
The parameters $m$ and $\tau$ (dimensions of the phase space and delay/lag between samples, respectively) can be deduced in a wide variety of ways, as described in [4],[5],[6]. For this work, we have used the False Nearest Neighbors method for deducing $m$ and the mutual information criterion for the choice of $\tau$.

The deduced parameters are presented in table 1 .

TABLE I

EMBEDDING PARAMETERS

\begin{tabular}{|c|c|c|c|}
\hline & PD & COMM & PD+COMM \\
\hline $\mathrm{m}$ & 8 & 8 & 8 \\
\hline$\tau$ & 30 & 10 & 30 \\
\hline
\end{tabular}

After properly embedding the signals in the corresponding phase space that completely unfolds their dynamics, we must use a tool that allows data visualization. Seeing as we are working in an 8-dimensional space, visual representation of the trajectories becomes impossible in a straightforward matter.

A powerful tool that is used in situations of this type is the "cosine distance" between vectors $u$ and $v$, expressed in equation (3):

$$
C D_{u v}=1-\cos \theta
$$

, where

$$
\cos \theta=\frac{u \cdot v}{\|u\| \cdot\|v\|}
$$

, where $\|\cdot\|$ is the Euclidean norm operator and $u \cdot v$ is the Euclidean dot product.

So, for an $M \times m$ trajectory matrix, the distance matrix will contain $M \times M$ points, which consist of the pair-wise cosine distance between the said pair of points.

The general advantages of using the cosine distance are:

- $\quad$ Bounded output: between 0 and 1;

- Commonly used in high-dimensional spaces;

- Also commonly used in information retrieval and text mining;

- Widely used measure of cohesion within clusters for data mining;

- Relatively simple to compute;

- Intuitive measure of distance/dissimilarity.

After computing the cosine distance matrices, we have normalized (0-to-1 scale) and threshold them (empirically chosen at 0.5 , in order to keep only the points that are further apart), thus obtaining binary matrices.

Figures 4-to-6 present the distance matrices of the partial discharge (PD), the communication signal (COMM) and of the COMM overlapping the PD, respectively. 


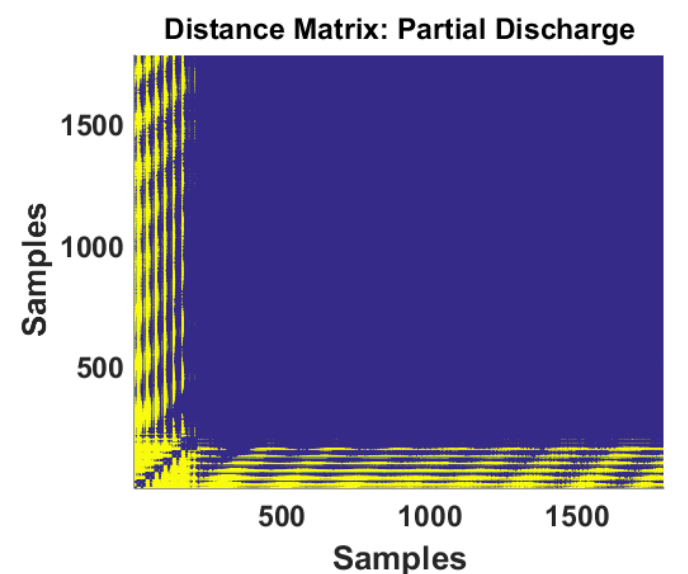

Figure 4. Cosine distance matrix for Partial Discharge.

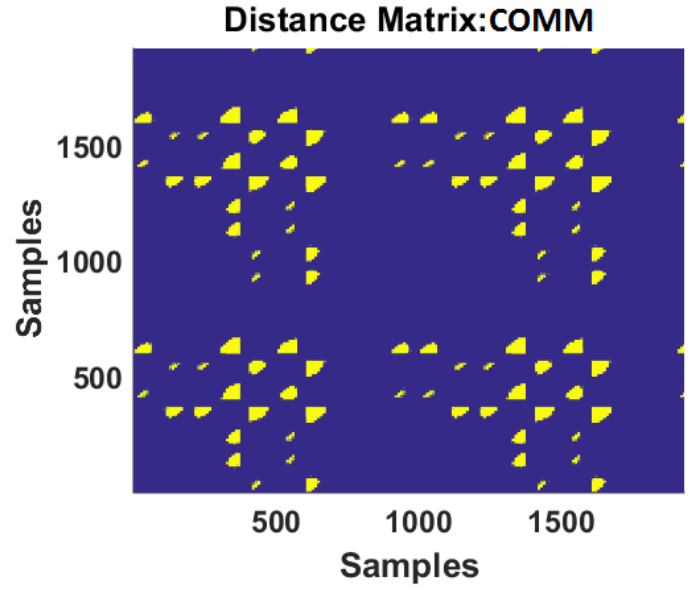

Figure 5. Cosine distance matrix for COMM signal.

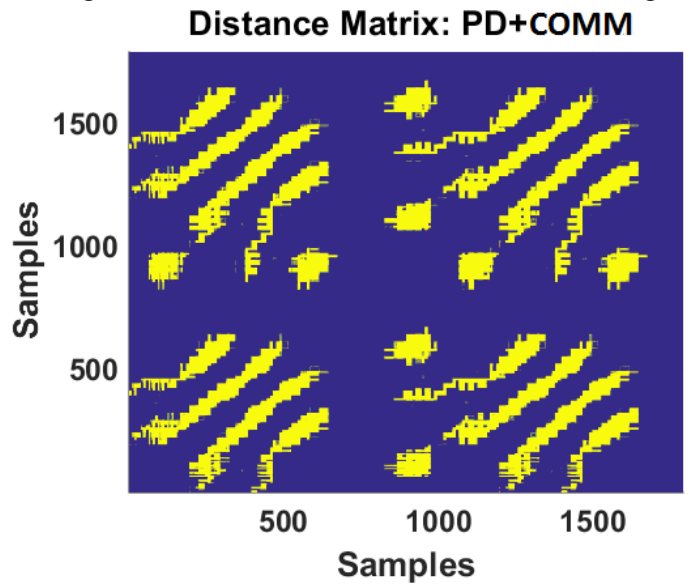

Figure 6. Cosine distance matrix for PD+COMM signal.

We can see in the above images, as in other measurements we have carried out, that in the case of the mixed partial discharge and COMM signal, the binary image that resulted from the thresholding of the cosine distance matrix, presents a great number of diagonal shapes, parallel to the principal diagonal of the matrix. To confirm this, we have used the Hough Transform, in order to investigate the presence of lines in the images and what are the orientations of these lines.

Lines can be represented in a 2D image (with coordinates $\mathrm{XY}$ ), in the following manner:

$$
y=a x+b
$$

where $x y$ are the coordinates of a pixel and $a$ and $b$ are parameters of that particular line.

However, the form expressed in equation (5) is not capable of representing vertical lines. Thus, a more proper form, used in the Hough Transform, is given in equation (6):

$$
r=x \cdot \cos \theta+y \cdot \sin \theta
$$

which can be rewritten in the form:

$$
y=-\frac{\cos \theta}{\sin \theta} \cdot x+\frac{r}{\sin \theta}
$$

where $r$ and $\theta$ are the distance from the line to the origin and the angle of line, respectively.

All lines can be represented in this form, as $\theta$ goes from 0 to 180 degrees and $r$ varies in $\mathbf{R}^{+}$.

Thus, the Hough space has two dimensions, $r$ and $\theta$, and a line is represented by a single point, corresponding to a unique set of parameters $\left(r_{u}\right.$ and $\left.\theta_{u}\right)$. The line-to-point mapping is illustrated in figure 7 and the mapping of a point to the Hough space is depicted in figure 8 .

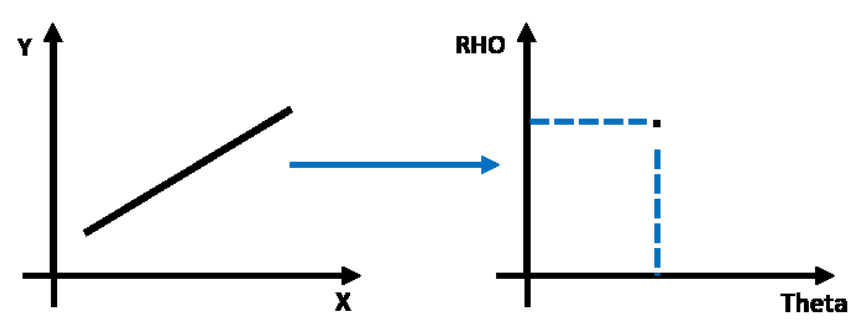

Figure 7. Line to point mapping to the Hough space.

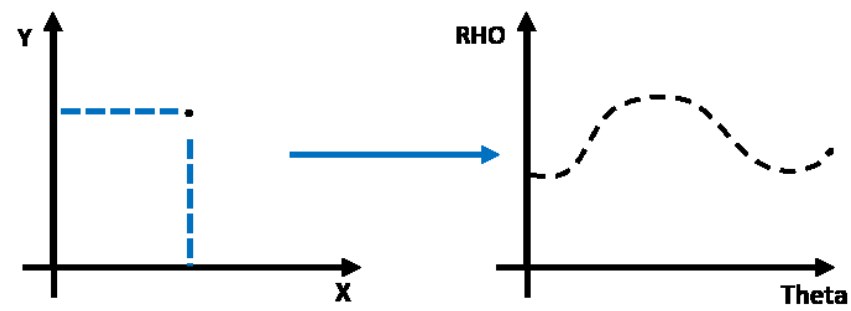

Figure 8. Point to line mapping to the Hough space.

Figure 8 shows how a point is mapped to all lines that can pass through that point, which results in a sine-line line in the Hough space.

Having established the suitability of the Hough Transform for line inspection, we have carried out this type of analysis for each of the three cases presented previously: partial discharge, COMM and mixed signal. Figures 9, 10 and 11 illustrate the results. 


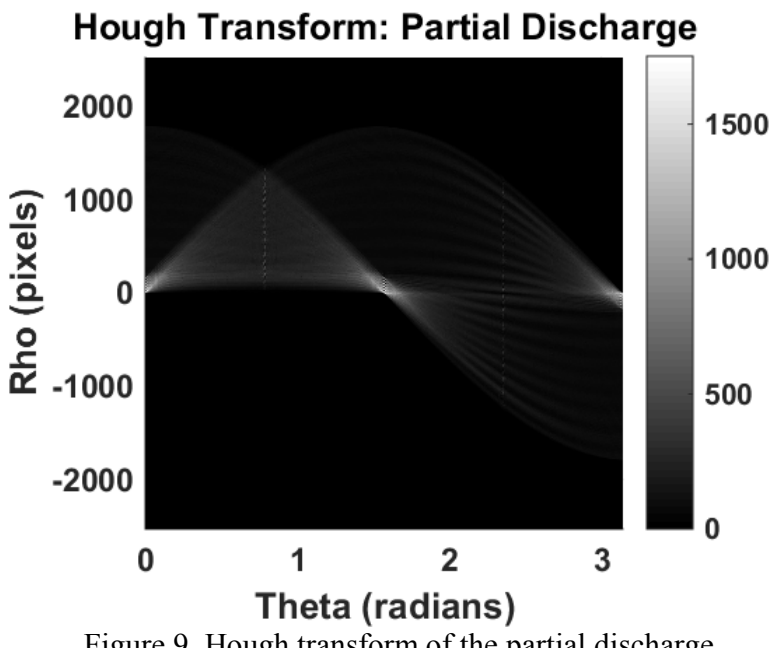

Figure 9. Hough transform of the partial discharge.

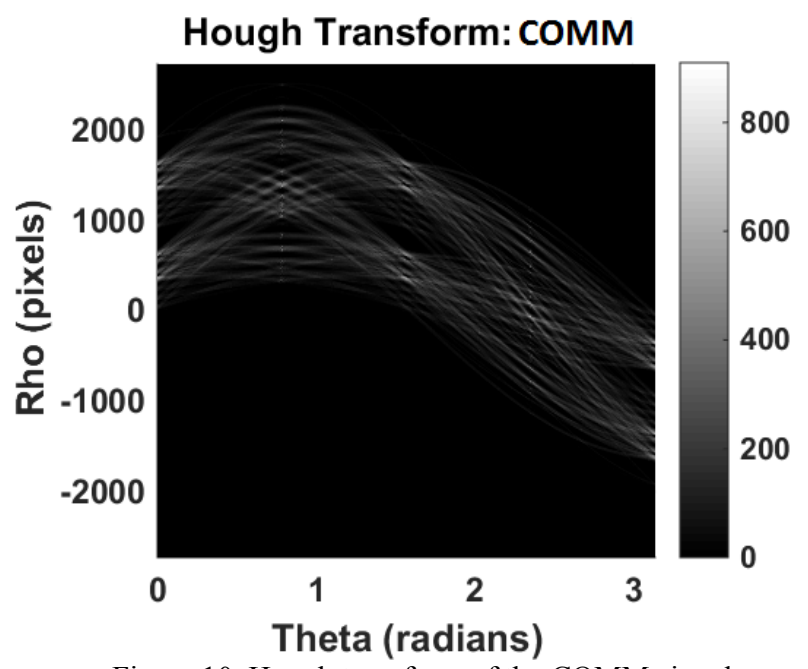

Figure 10. Hough transform of the COMM signal.

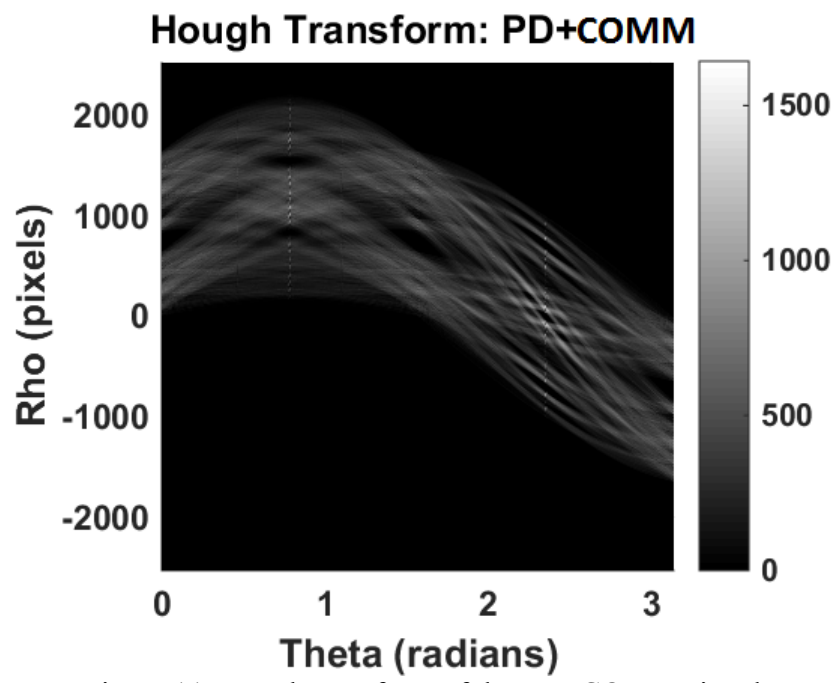

Figure 11. Hough transform of the PD+COMM signal.

The large concentration of lines for the $\mathrm{PD}+\mathrm{COMM}$ signal, around the $\pi / 4=0.7854\left(45^{\circ}\right) \theta$ point indicates a high number of lines parallel to the diagonal in figure 6 .

Hough-based diagonal line detection is depicted in figure 12. In our application, we focus directly on the largest $45^{\circ}$ line found in the binarized distance matrix.

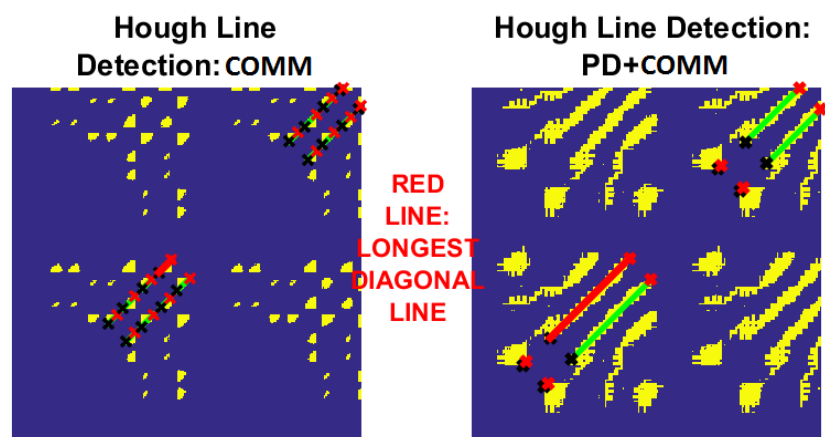

Figure 12. Hough-based Longest Diagonal Detection.

\section{RESUlTS \& CONCLUSION}

We have carried out this analysis for 16 acquired partial discharge signals that occurred during the several hours of functioning of a resistive load, in a DC network, which also included a COMM-based communication system. There is clear separation between the two classes (COMM and PD+COMM signals).

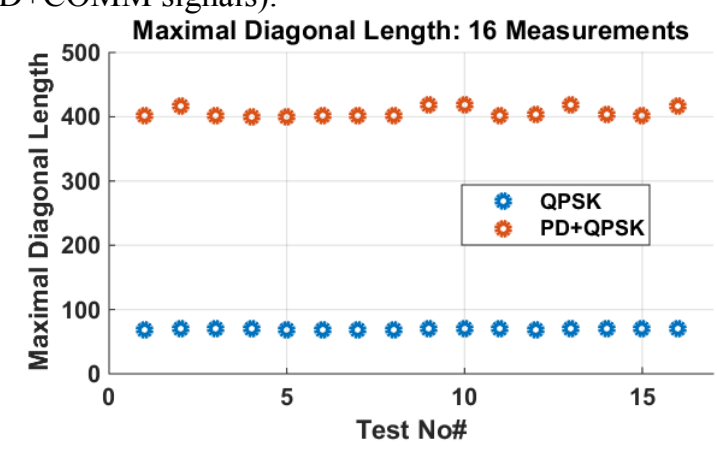

Figure 12. Results for $16 \mathrm{PD} / \mathrm{PD}+\mathrm{COMM}$ pairs of signals: maximal diagonal length.

The results are very promising and we intend to extend this study to a larger database of signals, as it enables the tackling of issues that are very difficult to solve in the temporal domain.

\section{REFERENCES}

[1] Boggs and G. C. Stone, "Fundamental limitations in the measurement of corona effect and partial discharges" Ontario Hydro Research, Toronto, Canada IEEE Transactions on Electrical Insulation Vol. EI-17 No.2, April 1982143.

[2] A. Krivda, "Automated Recognition of Partial Discharges", IEEE Transactions on Dielectrics and Electrical Insulation, Vol.2, No. 5, October 1995.

[3] I. Candel, B. Gottin, C. Ioana, "Monitoring Partial Discharges in Power Networks: contribution to the energetic distribution security", IEEE EnergyCon, 2010, DOI: 10.1109/ENERGYCON.2010.5771712.

[4] A. Digulescu, C. Bernard, E. Lungu, I. Candel, C. Ioana, G. Vasile, "Transient signal characterization using multi-lag phase space analysis", IEEE COMCAS, 2015, DOI: 10.1109/COMCAS.2015.7360411.

[5] I. Candel, A. Digulescu, C. Ioana, A. Serbanescu, E. Sofron, "Optimization of partial discharge detection in high voltage cables based on advanced signal processing techniques", IEEE ISSPA, 2012, DOI: 10.1109/ISSPA.2012.6310489.

[6] H. Abarbanel, "Analysis of Observed Chaotic Data", Springer, 1996, ISBN 978-0-387-98372-1.

[7] F. Takens (1981). "Detecting strange attractors in turbulence". Lecture Notes in Mathematics. pp. 366-381. 